\title{
Change in the neutrophil-to-lymphocyte ratio during chemotherapy may predict prognosis in patients with advanced or metastatic colorectal cancer
}

\author{
TETSUTARO NEMOTO, SHUNGO ENDO, NORIYUKI ISOHATA, DAISUKE TAKAYANAGI, \\ DAIKI NEMOTO, MASATO AIZAWA, KENICHI UTANO and KAZUTOMO TOGASHI
}

Department of Coloproctology, Aizu Medical Center, Fukushima Medical University, Fukushima 969-3492, Japan

Received November 29, 2020; Accepted March 1, 2021

DOI: $10.3892 / \mathrm{mco} .2021 .2269$

\begin{abstract}
The neutrophil-to-lymphocyte ratio (NLR) has been reported to be an independent prognostic factor of unresectable advanced or metastatic colorectal cancer (uCRC). However, few studies have documented changes in NLR during chemotherapy. The current study analyzed whether a change in NLR during chemotherapy in patients with uCRC could be used as a prognostic biomarker. The present retrospective study enrolled 71 patients who received first-line chemotherapy for uCRC between April 2012 and April 2019. The exclusion criteria were as follows: Acute infection or systemic inflammatory disease, duration of first-line chemotherapy $<3$ months, curative resection after chemotherapy and treatment with granulocyte-colony stimulating factor within 1 month. NLR, Lymphocyte-to-monocyte ratio (LMR), platelet-to-lymphocyte ratio (PLR), lactate dehydrogenase, alkaline phosphatase (ALP), albumin, carcinoembryonic antigen (CEA) and carbohydrate antigen 19-9 (CA19-9) levels were calculated before chemotherapy and at 3 months after chemotherapy. Among these laboratory data, NLR, PLR, ALP, CEA and CA19-9 levels were significantly decreased during chemotherapy. For Cox univariate analyses, these five data makers were divided into two groups: Decreased and increased (comparing before and at 3 months after chemotherapy). Only the change in NLR was significantly associated with overall survival $(\mathrm{P}=0.0002)$. Furthermore, the overall survival $(\mathrm{P}<0.0001)$ and progression-free survival $(\mathrm{P}=0.0041)$ of patients with decreased NLR was increased compared with patients with increased NLR. The change in
\end{abstract}

Correspondence to: Dr Shungo Endo, Department of Coloproctology, Aizu Medical Center, Fukushima Medical University, 21-2 Maeda, Tanisawa, Kawahigashi-machi, Aizuwakamatsu, Fukushima 969-3492, Japan

E-mail: endoswing@gmail.com

Key words: neutrophil-to-lymphocyte ration, inflammation-based indicators, colorectal cancer, chemotherapy, prognostic factor, overall survival, progression-free survival
NLR from pre-chemotherapy to 3 months following chemotherapy was determined to be a predictor of prognosis in patients with uCRC. The ability to predict prognosis at an early phase of chemotherapy may provide useful information for the selection of subsequent treatment and may improve the quality of patient life.

\section{Introduction}

Colorectal cancer (CRC) is the most common neoplasm in the gastrointestinal tract worldwide (1) and is considered to be have a good prognosis among the gastrointestinal cancers. However, the prognosis of unresectable colorectal cancer (uCRC) due to progression or metastasis is worse, and tools are required for predicting the disease course and first-line chemotherapy response.

Recently, there has been an argument supporting the role of inflammation in malignant tumor growth and progression (2). Emerging studies have demonstrated that inflammatory markers, such as the neutrophil-to-lymphocyte ratio (NLR) play substantial roles in the prediction of survival in different malignant tumors, including colorectal, breast, ovarian, gastric and bladder cancers (3-8). The researchers focused mainly on pretreatment inflammatory markers, while the dynamic changes in inflammatory markers after chemotherapy were not considered. Changes in inflammatory markers during chemotherapy might be a valuable tool to assess prognosis because chemotherapy may change the inflammatory response. However, even though changes in systemic inflammatory markers might dynamically reflect the modification of the balance between the host inflammatory response and the immune response against cancer during chemotherapy, their value is not fully understood.

NLR has been reported as an independent predictive factor for the prognosis of uCRC $(9,10)$. Relationships between cancer treatment outcomes and inflammation-based indicators, including NLR, platelet-to-lymphocyte ratio (PLR), lymphocyte-to-monocyte ratio (LMR), and modified Glasgow prognostic score, have been widely studied. Among these, the NLR is a representative index. An elevated NLR reflects greater systemic inflammation, which can induce cancer progression via the production of pro-inflammatory and angiogenic cytokines, and is associated with reduced tumor-specific 
immunity, including a reduced number of tumor-infiltrating lymphocytes in the tumor microenvironment $(11,12)$. Although NLR is considered a promising prognostic biomarker based on previous reports, it is unlikely to be used in clinical practice because there is no consensus on the cutoff value. A recent meta-analysis evaluating NLR as a prognostic biomarker in patients with CRC also noted this heterogeneity on the cutoff level as a critical limitation (13). Whereas, there are a few reports about the change in NLR during chemotherapy (6), examining the association between change in NLR and prognosis would obviate the need to set the cutoff level. This study aimed to explore the prognostic impact of the change in NLR during first-line chemotherapy on outcomes in patients with uCRC.

\section{Patients and methods}

Study population. This was a retrospective single-institutional study and included 71 patients who received first-line chemotherapy for uCRC between April 2012 and April 2019 at the Department of Coloproctology, Aizu Medical Center, Fukushima Medical University. The Fukushima Medical University ethics committee approved this study. Patients' characteristics data were collected from medical records, and all patients presented with histologically confirmed colorectal adenocarcinoma. The exclusion criteria were: i) Clinical confirmation of acute infection, systemic inflammation or other autoimmune disorders; ii) patients who received steroid therapy; iii) patients with hematologic disorders; iv) patients diagnosed with synchronous second malignancy arising from different regions; v) patients who were given first-line chemotherapy for less than 3 months; vi) patients who had undergone curative resection after chemotherapy and vii) patients who had been injected with granulocyte-colony stimulating factor (G-CSF) within one month.

Chemotherapy regimens. Chemotherapy was administered after the diagnosis of uCRC. First-line chemotherapy protocols were oxaliplatin-based, irinotecan-based or oxaliplatin plus irinotecan-based, and molecular targeted drugs were added to all cases based on $R A S$ status and by the discretion of the attending physician.

Tumor response and data collection. All patients underwent baseline computed tomography (CT) screening before chemotherapy, and follow-up imaging was performed every 3 months. The response evaluation criteria for solid tumors (RECIST) were used to evaluate radiological responses. Baseline characteristics included age, sex, performance status, tumor location, metastatic organs, histology, RAS status, blood cell count (white blood cells, neutrophils, lymphocytes, monocytes, platelets), albumin, alkaline phosphatase (ALP), lactate dehydrogenase (LDH), carcinoembryonic antigen (CEA), and carbohydrate antigen 19-9 (CA 19-9). Inflammation-based indicators, including NLR, LMR and PLR, and other laboratory data, including LDH, ALP, albumin, CEA, and CA 19-9, were obtained retrospectively, both before chemotherapy and 3 months after chemotherapy initiation. We analyzed the relationship between laboratory data that showed significant changes during chemotherapy and overall survival (OS) and progression-free survival (PFS).

Endpoints. The primary endpoint of this study was to determine whether a change in NLR is a prognostic factor for patients with uCRC treated with chemotherapy. The secondary endpoints were to examine the association of prognosis with the change in the index calculated from inflammatory markers such as LMR and PLR and the change in the tumor markers of colorectal cancer, such as CEA and CA 19-9.

Statistical analysis. Quantitative data were reported as median (range). All statistical analyses were performed using EZR (14). The Wilcoxon signed rank test was used to compare paired data, the Mann-Whitney U test was used to continuous variables, and the Chi-square tests (Fisher's exact tests or Pearson's Chi-square test) were used to compare discrete variables. To identify prognostic factors, Cox's proportional hazards model was used for univariate analyses. For univariate analyses, the laboratory data that changed significantly during chemotherapy were examined in two groups: Decrease and increase, and overall survival time was used as the time variable. OS and RFS analyses conducted using the Kaplan-Meier method and the log-rank test were used to determine the significance of the survival curves. $\mathrm{P}$-values $<0.05$ were considered statistically significant.

\section{Results}

The baseline characteristics of patients are summarized in Table I. In this study, the median follow-up period was 21.0 (5.1-73.4) months. The gender was predominantly male, and most of the patients were in good general condition with Eastern Cooperative Oncology Group (ECOG) performance scores of 0 to 1 in $98.6 \%$ of cases. In terms of tumor location, right-sided colon, left-sided colon, and rectum had almost the same proportions. The proportions of patients who previously received adjuvant chemotherapy and who had metachronous metastasis were similar. There were more cases of using an anti-VEGF antibody as a molecular-targeted drug in addition to chemotherapy, although there was an equal number of cases of wild type and mutant type in $R A S$ status.

The treatment characteristics of the patients are summarized in Table II. Chemotherapy was administered after the diagnosis of uCRC. First-line chemotherapy was oxaliplatin-based in 30 cases, irinotecan-based in 34 cases, and oxaliplatin plus irinotecan-based in 7 cases. Molecular targeted drugs included anti-VEGF antibody (bevacizumab) in 58 cases and anti-EGFR antibody (cetuximab or panitumumab) in 13 cases. The total number of chemotherapy regimens were as follows: One regimen, 19 cases; $2-4$ regimens, 42 cases; and 5-7 regimens, 10 cases. The best responses to chemotherapy were complete response (CR), 2 cases; partial response (PR), 30 cases; stable disease (SD), 28 cases; and progressive disease (PD), 11 cases. There were 10 patients (14.1\%) who received palliative resection of the primary lesion/metastatic lesions after chemotherapy induction.

Changes in inflammation-based indicators, other laboratory data, and tumor markers are shown in Table III. Significant changes between pre-chemotherapy and 3 months 
Table I. Baseline characteristics.

\begin{tabular}{|c|c|}
\hline Characteristic & $\mathrm{N}(\%)$ \\
\hline Follow-up period (months) ${ }^{\mathrm{a}}$ & $21.0(5.1-73.4)$ \\
\hline Age $\left(\right.$ years) ${ }^{\mathrm{a}}$ & $66(37-84)$ \\
\hline \multicolumn{2}{|l|}{ Sex } \\
\hline Male & $53(74.6)$ \\
\hline Female & $18(25.4)$ \\
\hline \multicolumn{2}{|l|}{ Performance score (ECOG) } \\
\hline 0 & $57(80.3)$ \\
\hline 1 & $15(18.3)$ \\
\hline 2 & 1 (1.4) \\
\hline \multicolumn{2}{|l|}{ Tumor location } \\
\hline Right & $22(31.0)$ \\
\hline Left & $49(69.0)$ \\
\hline \multicolumn{2}{|l|}{ Colon/rectum } \\
\hline Colon & $44(62.0)$ \\
\hline Rectum & $27(38.0)$ \\
\hline \multicolumn{2}{|c|}{ Primary lesion removal before chemotherapy } \\
\hline Yes & $48(67.6)$ \\
\hline No & $23(32.4)$ \\
\hline \multicolumn{2}{|l|}{ Adjuvant chemotherapy } \\
\hline Yes & $27(33.8)$ \\
\hline No & $47(66.2)$ \\
\hline \multicolumn{2}{|l|}{ Indication for chemotherapy } \\
\hline Unresectable primary lesion & $3(4.2)$ \\
\hline Unresectable local recurrence & $4(5.6)$ \\
\hline Distant metastasis & $66(93.0)$ \\
\hline \multicolumn{2}{|c|}{ Synchronous/metachronous metastasis } \\
\hline Synchronous & $40(56.3)$ \\
\hline Metachronous & $29(40.8)$ \\
\hline \multicolumn{2}{|l|}{ Metastasis sites } \\
\hline Liver & $38(53.5)$ \\
\hline Lung & $31(43.7)$ \\
\hline Lymph node & $17(23.9)$ \\
\hline Peritoneum/local & $14(19.7)$ \\
\hline \multicolumn{2}{|l|}{ Number of metastasis sites } \\
\hline 0 & $2(2.8)$ \\
\hline 1 & $39(54.9)$ \\
\hline$\geq 2$ & $30(42.2)$ \\
\hline \multicolumn{2}{|l|}{ Histology } \\
\hline Differentiated & $67(94.4)$ \\
\hline Non-differentiated & $4(5.6)$ \\
\hline \multicolumn{2}{|l|}{$R A S$ gene status } \\
\hline Wild & $31(43.7)$ \\
\hline Mutant & $31(43.7)$ \\
\hline Unknown & $9(12.7)$ \\
\hline
\end{tabular}

${ }^{a}$ Data are presented as the median (range). ECOG,Eastern Cooperative Oncology Group.

after initiation of chemotherapy were observed for NLR and PLR among the inflammation-based indicators, and LDH and
Table II. Treatment characteristics.

\begin{tabular}{lr}
\hline Treatment & $\mathrm{N}(\%)$ \\
\hline $\begin{array}{l}\text { First-line chemotherapy } \\
\text { Oxaliplatin based }\end{array}$ \\
Irrinotecan based & $30(42.3)$ \\
Oxaliplatin + Irrinotecan & $34(47.9)$ \\
Molecular targeted drugs & $7(9.9)$ \\
Anti-VEGF & \\
Anti-EGFR & $58(81.7)$ \\
Total number of regimens & $13(18.3)$ \\
1 & \\
$2-4$ & $19(26.8)$ \\
5-7 & $42(59.2)$ \\
Palliative surgery after chemotherapy & $10(14.1)$ \\
Yes & \\
No & $10(14.1)$ \\
Chemotherapy response & $61(85.9)$ \\
CR & \\
PR & $2(2.8)$ \\
SD & $30(42.3)$ \\
PD & $28(39.4)$ \\
\end{tabular}

EGFR, endothelial growth factor receptor; CR, complete response; $\mathrm{PR}$, partial response; $\mathrm{SD}$, stable disease; $\mathrm{PD}$, progressive disease.

ALP levels among other laboratory data. CEA and CA 19-9 as tumor markers showed significant changes. Among these factors, we analyzed NLR, PLR, ALP, CEA, and CA 19-9, which showed a significant difference in terms of change during chemotherapy, using Cox's univariate analyses (Table IV). Only the change in NLR was statistically significant with respect to change and prognosis pre-chemotherapy and 3 months after the initiation of chemotherapy.

Based on these results, we considered the NLR prechemotherapy and 3 months after the initiation of chemotherapy to be a prognostic factor for patients with uCRC and conducted our analysis. Table $\mathrm{V}$ shows the relationship between clinicopathologic factors in the two groups: There were 61 cases with a decrease in NLR and 10 cases with an increase in NLR during this period. Statistically significant differences were seen in the indications of chemotherapy. Table VI shows the relationship between the chemotherapeutic factors with NLR decrease and increase groups, there was a significant different only in the number of total chemotherapy regimens. In addition, OS and PFS were analyzed. OS was significantly better for patients with a decreased NLR than for those with an increased NLR $(\mathrm{P}<0.0001)$, with median survival times of 26.6 months and 10.1 months, respectively (Fig. 1), whereas PFS was 13.6 months and 5.2 months $(\mathrm{P}=0.0041)$, respectively (Fig. 2).

\section{Discussion}

CRC is considered to have a good prognosis among the gastrointestinal cancers. However, the prognosis of uCRC due to 
Table III. Changes in inflammation-based indicators, other laboratory data and tumor markers.

\begin{tabular}{llrr}
\hline Marker & Pre-chemotherapy, median (range) & 3 months after chemotherapy, median (range) & P-value $^{\mathrm{a}}$ \\
\hline NLR & $2.7(1.2-9.8)$ & $1.6(0.5-14.1)$ & $<0.0001$ \\
LMR & $4.0(0.8-13.3)$ & $4.0(0.6-10.2)$ & 0.9247 \\
PLR & $159.6(69.6-504.9)$ & $123.1(50.3-823.5)$ & $<0.0001$ \\
LDH $(\mathrm{IU} / \mathrm{l})$ & $216.0(118.0-4068.0)$ & $212.0(112.0-1732.0)$ & 0.0077 \\
ALP $(\mathrm{IU} / \mathrm{l})$ & $282.0(139.0-5561.0)$ & $261.0(37.4-1,297.0)$ & 0.0003 \\
ALB $(\mathrm{g} / \mathrm{dl})$ & $3.7(2.1-4.8)$ & $7.7(2.4-4.6)$ & 0.9541 \\
CEA $(\mathrm{ng} / \mathrm{ml})$ & $21.8(1.7-22527.0)$ & $20.8(0.6-23868.0)$ & $<0.0001$ \\
CA19-9 $(\mathrm{U} / \mathrm{ml})$ & $40.9(0-16926.0)$ & 0.0001
\end{tabular}

${ }^{\mathrm{a} A}$ Wilcoxon signed rank test was used to compare continuous variables. NLR, neutrophil-to-lymphocyte ratio; LMR, lymphocyte-to-monocyte ratio; PLR, platelet-to-lymphocyte ratio; LDH, lactate dehydrogenase; ALP, alkaline phosphatase; ALB, albumin; CEA, carcinoembryonic antigen; CA19-9, carbohydrate antigen 19-9.

Table IV. Cox's univariate analyses for changes in NLR, PLR, ALP, CEA and CA 19-9.

\begin{tabular}{lccc}
\hline Marker & Hazard ratio $^{\mathrm{a}}$ & $95 \%$ CI & P-value \\
\hline NLR & 3.950 & $1.937-8.054$ & 0.0002 \\
PLR & 1.325 & $0.716-2.453$ & 0.3703 \\
ALP (IU/l) & 0.755 & $0.428-1.332$ & 0.3318 \\
CEA $(\mathrm{ng} / \mathrm{ml})$ & 1.186 & $0.659-2.134$ & 0.5695 \\
CA19-9 $(\mathrm{U} / \mathrm{ml})$ & 1.173 & $0.673-2.043$ & 0.5740 \\
\hline
\end{tabular}

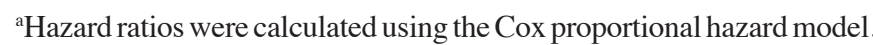
NLR, neutrophil-to-lymphocyte ratio; PLR, platelet-to-lymphocyte ratio; ALP, alkaline phosphatase; CEA, carcinoembryonic antigen; CA19-9, carbohydrate antigen 19-9; CI, confidence interval.

progression or metastasis is poor. Although uCRC is treated with multidisciplinary therapy, mainly chemotherapy, its survival rate has not reached the desired level. CEA and CA 19-9 are considered useful markers in the treatment of colorectal cancer, but they are highly variable and not accurate enough to be recognized as prognostic markers for patients with uCRC treated with chemotherapy. Therefore, the need for predictive and prognostic markers during chemotherapy for $\mathrm{UCRC}$ is increasing.

In this study, we examined the NLR, LMR, and PLR, which have been reported to be useful as inflammatory markers, but only the NLR and PLR showed a significant difference between prechemotherapy and 3 months after chemotherapy initiation. Finally, only NLR correlated with prognosis. Patients were divided into two groups: Decreased NLR and increased NLR, and their clinicopathological factors were compared. However, there was no difference in the response to chemotherapy, but there was a difference in the total number of chemotherapy regimens. There was also a significant difference in the CEA and CA 19-9 level between prechemotherapy and 3 months after the initiation of chemotherapy, but this did not correlate with prognosis.

The NLR has been suggested as a prognostic marker in various solid tumors $(3-8,15,16)$. NLR is a factor related to systemic inflammation, which is associated with cancer growth. Systemic inflammation may lead to tumor initiation through genetic mutations, genomic instability, and epigenetic modifications. Inflammation promotes tissue repair responses that induce the proliferation of premalignant cells and increase their viability. It is also involved in angiogenesis, immunosuppression, inhibition of apoptosis, and DNA damage, ultimately and contributing to metastatic spread $(12,17)$. A high NLR indicates a relatively elevated neutrophil count and depressed lymphocyte count. Neutrophils are thought to produce vascular endothelial growth factor and various matrix proteases (18).

Another promising application of inflammatory-based scores as predictive biomarkers is a longitudinal change before and after treatment. A similar previous study in patients with metastatic gastric cancer reported that constantly elevated NLR or an increase in NLR during chemotherapy correlated with poor OS, PFS, and chemotherapy response (19). There was study compared the preoperative NLR and the differences in NLR preoperative and one-month postoperative by determine the cutoff value from receiver operating characteristic (ROC) curve analysis, respectively, with prognosis in colorectal cancer resection cases (20). That study reported better OS and disease-free survival (DFS) when the preoperative NLR was low and when the NLR was reduced by resection. Another similar study also reported that when preoperative NLR was low and 7 days postoperative NLR was low, and when preoperative NLR was high and postoperative NLR was low in colorectal cancer surgical treatment, OS and PFS were better (21). Those studies needed cutoff value determined by ROC curve analysis that was cumbersome. Contrarily, a study examining changes in NLR with uCRC patients before and after two cycles of chemotherapy (FOLFIRI + bevacizumab) reported that an increase NLR led to significantly longer OS than a decrease NLR, in patients with SD (22). That result was a different from our study in the manner that they were shorter period which evaluate the change in NLR and were limited to SD patients.

Divided of the patients into the NLR decrease and increase groups resulted in significant differences in OS and PFS. There was also a significant difference in the number of chemotherapy regimens. Furthermore, the large differences between the two groups in terms of both OS and PFS suggest that patients with an increased NLR may have had rapid cancer progression that 
Table V. Relationship between NLR and clinicopathologic factors.

\begin{tabular}{|c|c|c|c|}
\hline Clinicopathologic factor & NLR decrease $(n=61)$ & NLR increase $(n=10)$ & P-value \\
\hline Age, years; median (range) & $65(32-84)$ & $68(58-80)$ & 0.5905 \\
\hline Sex & & & 0.4493 \\
\hline Male & 47 & 6 & \\
\hline Female & 14 & 4 & \\
\hline Performance status & & & 0.1901 \\
\hline 0 & 51 & 6 & \\
\hline $1-2$ & 10 & 4 & \\
\hline Tumor location & & & 0.7671 \\
\hline Right & 18 & 4 & \\
\hline Left & 43 & 6 & \\
\hline Colon/rectum & & & $>0.9999$ \\
\hline Colon & 38 & 6 & \\
\hline Rectum & 23 & 4 & \\
\hline Primary lesion removal before chemotherapy & & & $>0.9999$ \\
\hline Yes & 41 & 7 & \\
\hline No & 20 & 3 & \\
\hline Adjuvant chemotherapy & & & 0.4193 \\
\hline Yes & 19 & 5 & \\
\hline No & 42 & 5 & \\
\hline Indication of chemotherapy & & & 0.2886 \\
\hline Unresectable primary lesion or local recurrence & 3 & 2 & \\
\hline Unresectable metastasis & 58 & 8 & \\
\hline Synchronous/metachronous metastasis & & & 0.8369 \\
\hline Synchronous & 35 & 5 & \\
\hline Metachronous & 24 & 5 & \\
\hline \multicolumn{4}{|l|}{ Metastasis site } \\
\hline Liver & 34 & 4 & 0.5600 \\
\hline Lung & 26 & 5 & 0.9267 \\
\hline Lymph node & 16 & 1 & 0.4746 \\
\hline Peritoneum/local & 11 & 3 & 0.6506 \\
\hline Number of metastasis sites & & & 0.6164 \\
\hline $0-1$ & 34 & 7 & \\
\hline$\geq 2$ & 27 & 3 & \\
\hline Histology & & & 0.1658 \\
\hline Differenciated & 59 & 8 & \\
\hline Non-differenciated & 2 & 2 & \\
\hline RAS gene status & & & 1 \\
\hline Wild & 27 & 4 & \\
\hline Mutant & 27 & 4 & \\
\hline
\end{tabular}

NLR, neutrophil-to-lymphocyte ratio.

would have made the continuation of chemotherapy difficult. These results may also indicate that patients with an increased NLR during chemotherapy have a systemic poor immunologic condition that does not improve with chemotherapy. An advanced state of so-called cancer cachexia is assumed. Although these results may be limited to the early period of chemotherapy, a study of patients undergoing late-line chemotherapy treated with
TAS-102, which is an oral combination of trifluridine and tipiracil, also reported that the NLR before initiation of treatment was significantly negatively associated with OS and PFS (23). The relationship between changes in NLR and prognosis should also be confirmed in late-line chemotherapy patients.

In this study, dynamic changes in NLR statistically correlated with OS and PFS. Therefore, we believe that the change in 
Table VI. Relationship between NLR and chemotherapeutic factors.

\begin{tabular}{|c|c|c|c|}
\hline Chemotherapeutic factor & NLR decrease $(\mathrm{n}=61)$ & NLR increase $(n=10)$ & P-value \\
\hline First-line chemotherapy & & & $0.8535^{\mathrm{a}}$ \\
\hline Oxaliplatin & 30 & 4 & \\
\hline Irinotecan & 25 & 5 & \\
\hline Oxaliplatin + Irinotecan & 6 & 1 & \\
\hline Molecular targeted drug (first-line chemotherapy) & & & $0.7703^{\mathrm{a}}$ \\
\hline Anti-VEGF & 49 & 9 & \\
\hline Anti-EGFR & 12 & 1 & \\
\hline Number of total regimens, median (range) & $3(1-7)$ & $1(1-4)$ & $0.0240^{\mathrm{b}}$ \\
\hline Palliative surgery after chemotherapy & & & $0.3297^{\mathrm{a}}$ \\
\hline Yes & 10 & 0 & \\
\hline No & 51 & 10 & \\
\hline Response to chemotherapy & & & $0.3077^{\mathrm{a}}$ \\
\hline $\mathrm{CR}$ & 2 & 0 & \\
\hline PR & 28 & 2 & \\
\hline $\mathrm{SD}$ & 23 & 5 & \\
\hline $\mathrm{PD}$ & 8 & 3 & \\
\hline $\mathrm{CR}+\mathrm{PR}+\mathrm{SD}$ vs. $\mathrm{PD}$ & 53 vs. 8 & 7 vs. 3 & $0.3700^{\mathrm{a}}$ \\
\hline
\end{tabular}

${ }^{a}$ Chi-square (Fisher's exact or Pearson's Chi-square) and ${ }^{b}$ Mann-Whitney U testes were used to compare discrete and continuous variables, respectively. NLR, neutrophil-to-lymphocyte ratio; EGFR, endothelial growth factor receptor; CR, complete response; PR, partial response; $\mathrm{SD}$, stable disease; $\mathrm{PD}$, progressive disease.

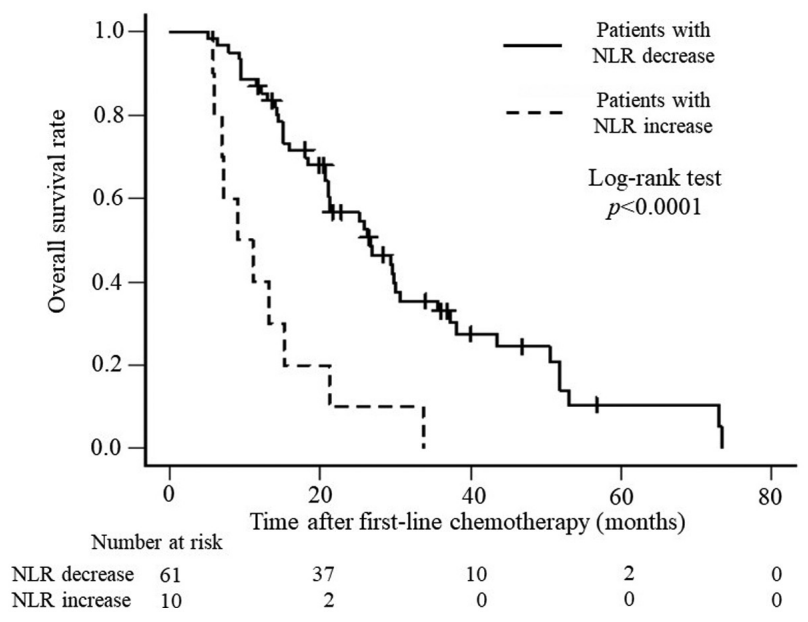

Figure 1. Kaplan-Meier curves presenting overall survival rates in at risk patients with increased or decreased NLR levels. NLR, neutrophil-tolymphocyte ratio.

NLR during chemotherapy may be more accurate in predicting prognoses after chemotherapy than the NLR calculated before chemotherapy. The ability to predict prognosis at an early phase after the introduction of chemotherapy will provide useful information for the selection of subsequent treatments and may contribute towards improving patients' quality of life. It should also be emphasized that the ROC curve analysis does not need to be used to determine the cutoff value, which makes it easier to determine.

The limitations of this study are its retrospective observational nature, small sample size, and its setting in a

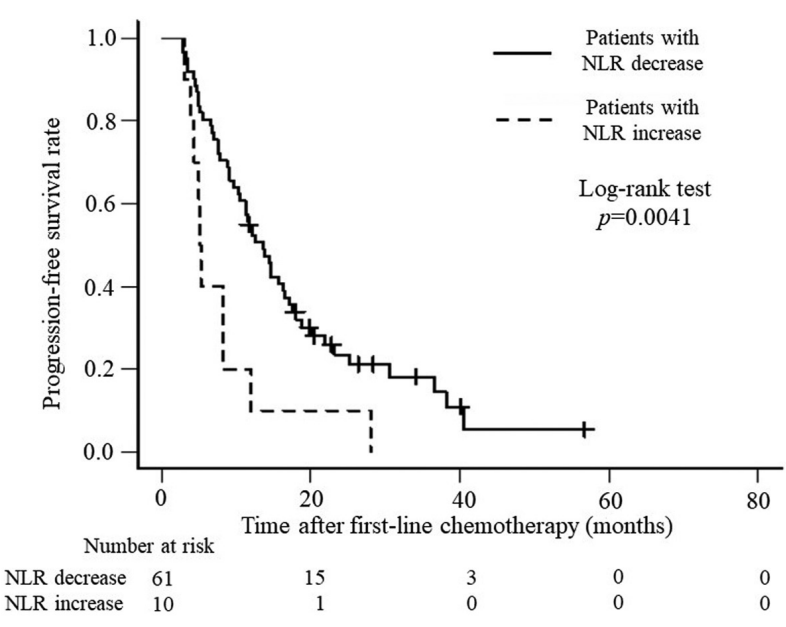

Figure 2. Kaplan-Meier curves presenting progression-free survival rates in at risk patients with increased or decreased NLR levels. NLR, neutrophil-to-lymphocyte ratio.

single institute in Japan, non-exclusion of some factors affecting NLR values. Although many studies have found the NLR to be useful as a prognostic biomarker, the cutoff level for NLR has been calculated using the median value of NLR or using receiver operating characteristic curve analyses, which may be difficult to use in routine clinical practice. For this reason, we recommend that the results of this study be validated prospectively without the need to establish a cutoff value. Further high-quality studies with larger cohorts are required to confirm this finding.

In conclusions, although OS and PFS correlated with changes in NLR prechemotherapy and 3 months after the 
initiation of chemotherapy, only the number of chemotherapy regimens showed an association between changes in NLR in clinicopathological factors. The ability to predict prognosis at an early phase after the introduction of chemotherapy will provide useful information for the selection of subsequent treatment protocols and may contribute toward the improvement of patients' quality of life.

\section{Acknowledgements}

Not applicable.

\section{Funding}

No funding was received.

\section{Availability of data and materials}

The datasets used and/or analyzed during the current study are available from the corresponding author on reasonable request.

\section{Authors' contributions}

SE designed the study. TN, SE, NI, DT, DN, MA and KU acquired the data. TN and SE confirm the authenticity of all the raw data. TN, KT and SE analyzed and interpreted the data and drafted the manuscript. SE and KT performed critical revision of the manuscript. SE supervised the study. All authors read and approved the final manuscript.

\section{Ethics approval and consent to participate}

The present study was approved by the Medical Ethics Committee of the Fukushima Medical University (approval no. General 2020-198). Written informed consent was not obtained due to the retrospective nature of the study.

\section{Patient consent for publication}

Not applicable.

\section{Competing interests}

The authors declare that they have no competing interests.

\section{References}

1. Jemal A, Bray F, Center MM, Ferlay J, Ward E and Forman D: Global cancer statistics. CA Cancer J Clin 61: 69-90, 2011.

2. Mantovani A, Allavena P, Sica A and Balkwill F: Cancer-related inflammation. Nature 454: 436-444, 2008.

3. Li MX, Liu XM, Zhang XF, Zhang JF, Wang WL, Zhu Y, Dong J, Cheng JW, Liu ZW, Ma L and Lv Y: Prognostic role of neutrophil-to-lymphocyte ratio in colorectal cancer: A systematic review and meta-analysis. Int J Cancer 134: 2403-2413, 2014

4. Pistelli M, De Lisa M, Ballatore Z, Caramanti M, Pagliacci A, Battelli N, Ridolfi F, Santoni M, Maccaroni E, Bracci R, et al Pre-treatment neutrophil to lymphocyte ratio may be a useful tool in predicting survival in early triple negative breast cancer patients. BMC Cancer 15: 195, 2015
5. Nomelini RS, Carrijo Chiovato AF, Abdulmassih FBF, da Silva RC, Tavares-Murta BM and Murta EFC: Neutrophil-to-lymphocyte ratio and platelet count as prognostic factors in ovarian malignancies. J Cancer Res Ther 15: 1226-1230, 2019.

6. Aizawa M, Gotohda N, Takahashi S, Konishi M and Kinoshita T: Predictive value of baseline neutrophil/lymphocyte ratio for T4 disease in wall-penetrating gastric cancer. World J Surg 35: 2717-2722, 2011.

7. Dirican A, Kucukzeybek Y, Somali I, Erten C, Demir L, Can A, Bahriye Payzin K, Vedat Bayoglu I, Akyol M, Koseoglu M, et al: The association of hematologic parameters on the prognosis of patients with metastatic renal cell carcinoma. J BUON 18: 413-419, 2013.

8. Kang M, Jeong CW, Kwak C, Kim HH and Ku JH: Preoperative neutrophil-lymphocyte ratio can significantly predict mortality outcomes in patients with non-muscle invasive bladder cancer undergoing transurethral resection of bladder tumor. Oncotarget 8: 12891-12901, 2017

9. Tsai PL, Su WJ, Leung WH, Lai CT and Liu CK: Neutrophil-lymphocyte ratio and CEA level as prognostic and predictive factors in colorectal cancer: A systematic review and meta-analysis. J Cancer Res Ther 12: 582-589, 2016.

10. Kishi Y, Kopetz S, Chun YS, Palavecino M, Abdalla EK and Vauthey JN: Blood neutrophil-to-lymphocyte ratio predicts survival in patients with colorectal liver metastases treated with systemic chemotherapy. Ann Surg Oncol 16: 614-622, 2009.

11. Chen ZY, Raghav K, Lieu CH, Jiang ZQ, Eng C, Vauthey JN Chang GJ, Qiao W, Morris J, Hong D, et al: Cytokine profile and prognostic significance of high neutrophil-lymphocyte ratio in colorectal cancer. Br J Cancer 112: 1088-1097, 2015.

12. Coussens LM and Werb Z: Inflammation and cancer. Nature 420: 860-867, 2002.

13. Malietzis G, Giacometti M, Kennedy RH, Athanasiou T, Aziz O and Jenkins JT: The emerging role of neutrophil to lymphocyte ratio in determining colorectal cancer treatment outcomes: A systematic review and meta-analysis. Ann Surg Oncol 21: 3938-3946, 2014.

14. Kanda Y: Investigation of the freely available easy-to-use software 'EZR' for medical statistics. Bone Marrow Transplant 48: 452-458, 2013.

15. Lee DY, Hong SW, Chang YG, Lee WY and Lee B: Clinical significance of preoperative inflammatory parameters in gastric cancer patients. J Gastric Cancer 13: 111-116, 2013.

16. Nagasaki T, Akiyoshi T, Fujimoto Y, Konishi T, Nagayama S, Fukunaga $Y$ and Ueno $M$ : Prognostic impact of neutrophil-to-lymphocyte ratio in patients with advanced low rectal cancer treated with preoperative chemoradiotherapy. Dig Surg 32: 496-503, 2015.

17. Grivennikov SI, Greten FR and Karin M: Immunity, inflammation, and cancer. Cell 140: 883-899, 2010.

18. Balkwill F and Mantovani A: Inflammation and cancer: Back to virchow? Lancet 357: 539-545, 2001.

19. Bozkurt O, Firat ST, Dogan E, Cosar R, Inanc M and Ozkan M: The prognostic value of the change in neutrophil-to-lymphocyte ratio during first-line palliative chemotherapy in patients with metastatic gastric cancer: A retrospective study. J BUON 24: 1992-1999, 2019

20. Guo D, Han A, Jing W, Chen D, Jin F, Li M, Kong L and Yu J: Preoperative to postoperative change in neutrophil-to-lymphocyte ratio predict survival in colorectal cancer patients. Future Oncol 14: 1187-1196, 2018.

21. CuiM,XuRand Yan B: A persistenthighneutrophil-to-lymphocyte ratio predicts poor prognosis in patients with colorectal cancer undergoing resection. Mol Clin Oncol 13: 63, 2020.

22. Formica V, Luccchetti J, Cunningham D, Smyth EC, Ferroni P, Nardecchia A, Tesauro M, Cereda V, Guadagni F and Roselli M: Systemic inflammation, as measured by the neutrophil/lymphocyte ratio, may have differential prognostic impact before and during treatment with fluorouracil, irinotecan and bevacizumab in metastatic colorectal cancer patients. Med Oncol 31: 166, 2014

23. Matsuda A, Yamada T, Matsumoto S, Sakurazawa N, Kawano Y, Shinozuka E, Sekiguchi K, Suzuki H and Yoshida H: Pretreatment neutrophil-to-lymphocyte ratio predicts survival after TAS-102 treatment of patients with metastatic colorectal cancer. Anticancer Res 39: 4343-4350, 2019. 